\title{
HOMOLOGACION DE SENTENCIA EXTRANJERA
}

\author{
ENRIQUE VESCOVI \\ Profesor de la Facultad de Derecho \\ y Ciencias Sociales de Montevideo
}

\section{I - PROPOSITO DE ESTA COLABORACION}

El tema de la homologación de la sentencia extranjera es uno de los que ha dado lugar en los últimos tiempos a una mas abundante literatura en materia de Derecho Internacional Privado $v$ en la de Procesal. Inclusive ha sido objeto de consideración en diversos Congresos Internacionales. Volverlo a plantear representaría una pérdida de tiempo inútil (1). Por consiguiente me propongo efectuar esta colaboración señalando algunos aspectos generales de interés $y$, especialmente, ilustrando a los miembros de esta reunión sobre la posición de la legislación, la doctrina y la jurisprudencia en nuestro medio. Considero que, con respecto a estos temas, en los cuales es necesaria una colaboración internacional, si no la unificación de legislaciones o de procedimientos, la primera etapa, para lo cual todavía en América nos encontramos muy distantes, debe ser la de conocer precisamente la situación de los diversos problemas en cáda país, punto de partida indispensable para lograr soluciones o principios comunes.

\section{II - Planteo del PROblema de la ejecucion de Sentencia EXTRANJERA}

\section{A) Tema a considerar.}

Según uno de nuestros mas destacados procesalistas el tema de la sentencia extranjera puede dividirse en los siguientes capítulos: a) naturaleza de la sentencia extranjera; b) eficacia de la sentencia extranjera; c) naturaleza de la acción que ejercitan las partes para obtener su reconocimiento; d) naturaleza de la sentencia de reconocimiento o de exequatur (2). 
De estos temas el sometido a nuestra consideración, está vinculado exclusivamente al último, o sea: "Homologación de la sentencia extranjera" al que nos referiremos, y que excede la simple cuestión de la "naturaleza".

\section{B) Distinciones metodológicas.}

Aún sin aceptar su validez no podemos dejar de reconocer que deben hacerse algunas distinciones con respecto al tema, que nos serán de utilidad en nuestra exposición. Mencionaremos las siguien. tes:

1) Materia del exequatur: sentencia, laudo arbitral, procedimientos de jurisdicción voluntaria.

Aunque el tema se refiera exclusivamente a la sentencia, es evidente que puede ser materia del exequatur no solo la sentencia $\epsilon$.h tranjera, propiamente dicha, sino también el laudo arbitral y los actos de jurisdicción voluntaria. Y todavía en el primer caso cabría la distinción entre la sentencia dictada por un Tribunal Judicial, propia. mente dicho, y la "sentencia" administrativa.

En general resulta diferente la solución respecto de estos temas, por lo cual nos abocamos exclusivamente a tratar la ejecución de la sentencia extranjera, sin perjuicio de que, al mencionar las soluciones del derecho positivo, nos refiramos también a estas otras decisiones.

2) Eficacia de la sentencia; distinción según los aspectos de la sentencia a ejecutar.

Los distintos autores y legislaciones suelen considerar tres aspectos distintos:

a) Fuerza ejecutiva de la sentencia;

b) Valor de cosa juzgada de la sentencia; y

c) Valor de la sentencia como documento (probatorio).

Sin perjuicio de señalar que, desde el punto de vista doctrinario tal distinción parece inadecuada, la recogemos porque la jurisprudencia, y, lo que es mas importante, la legislación, actúan en forma distinta, en el procedimiento del exequatur, según se trate de alguno de estos tres efectos, porque ella es aceptada por casi todos los internacionalistas (3).

Decimos que, en realidad, desde el punto de vista procesal, no cabría hacer una distinción, porque en todo caso se trata del problema de la eficacia de la sentencia. Lo esencial es que nos encontramos frente a un fallo extranjero que decide la cuestión y respecto del cual, para hacerlo valer, en cualquier circunstancia, debemos efectuar un pronunciamiento, que implique su reconocimiento por nuestra justicia (4). 
Parece indiscutible que, aún cuando se trate del efecto considerado menos importante (si podemos hacer una gradación cuantitativa en este problema que no es de cantidad), su valor probatorio, la aceptación de dicha sentencia implica no sólo reconocer la existencia de un documento, sino la validez de un juicio que termina con un fallo. Por esto parece más conveniente la solución del nuevo Código Italiano que, en su artículo 796 y siguientes, se refiere, no a la ejecución de la sentencia sino a su eficacia general (5).

3) Sentencia dictada por un Organo competente en materia internacional, o por un Organo competente en materia nacional (6).

Diversos tratados-bilaterales o regionales - distribuyen la competencia entre los diversos países. A veces pues el Juez nacional que ha dictado la sentencia tiene una competencia reconocida legalmente por los demás estados, (internacional) y habrá que atenerse a los tratados, para su ejecución. En otros casos, la sentencia a ejecutarse es dictada simplesmente por un Juez extranjero, competente en su esfera (nacional) y es el estado requerido para ejecutarla, quien regula libremente los requisitos exigidos para el exequatur, generalmente por normas contenidas en sus códigos de procedimientos.

\section{III - SOLUCIONES DE LOS TRATADOS DE MONTEVIDEO Y DEL DERECHO POSITIVO URUGUAYO.}

\section{1) Los Tratados de Montevideo.}

Varios países latinoamericanos, incluyendo el Uruguay, están ligados por distintos tratados de Derecho Internacional Privado, los llamados Tratados de Montevideo de 1889 (reformados en 1940) (7). En ellos se establecen disposiciones sobre la competencia de los jueces en materia internacional, sobre diversas materias, y reglas relativas a la ejecución de las sentencias, declarándose que éstas - y los fallos arbitrales - "tendrán en los territorios de los demás la misma fuerza que en el país donde fueron pronunciados"... si reúnen ciertos requisitos mínimos (art. 5).

En consecuencia y de acuerdo a la distinción que efectuáramos inicialmente, cuando se trata de ejecutar una sentencia dictada por un Juez competente en la esfera internacional, debemos atenernos a las disposicions del Tratado. De acuerdo con el mismo, las diferencias, existentes entre la sentencia en cuanto su cumplimiento o ejecución propriamente dicha $y$ en el caso de que se haga valer como prueba ("la autoridad de cosa juzgada de una sentencia o un fallo"), quedan borradas luego porque el procedimiento es prácticamente idéntico (Art. 7, 8 y 9 del Tratado de Derecho Internacional Procesal de 1940). La diferencia era mayor en el Tratado de 1889, pero el nuevo tratado si bien distinguió los dos casos (Arts. 7 y 9) luego - quizás por las observaciones doctrinarias hechas en las sesiones (8) - borró casi todas las diferencias en el procedimiento de ejecución. 
El Tratado de 1940 exige para la ejecución de la sentencia extranjera que ésta cumpla los siguientes requisitos:

a) Que hayan sido dictadas por un Tribunal competente en la esfera internacional. En esto sigue el principio ya establecido y el juicio de reconocimiento de la sentencia, debe empezar por determinar si el Juzgado que lo dictó era competente en la esfera internacional, esto es, de acuerdo con las normas de fondo dictadas por el propio Tratado.

b) Que tengan el carácter de ejecutoriados o pasados en autoridad de cosa juzgada en el Estado en donde hayan sido pronunciados.

Es otro de los requisitos exigidos, generalmente, por todas las leyes, tratados, doctrina y jurisprudencia.

c) Que la parte contra la cual se hubieran dictado haya sido legalmente citada, y representada o declarada rebelde, conforme a la ley del país en donde se siguió el juicio.

d) Que no se oponga al orden público del país en su cumplimiento.

Existe, pues, un juicio de exequatur, que consiste en la comprobación, por el Juzgado correspondiente, con audiencia del Ministerio Público y previa presenteción de los documentosi indispensables (Art. 6), del cumplimiento de dichos requisitos y en este caso los jueces y Tribunales, cuando se trata de hacer valer como prueba la sentencia, "se pronunciarán sobre su mérito en la sentencia que dicten" (Art. 9) y, cuando se trata de "ejecución", ordenarán "su cumplimiento por la vía que corresponda, de acuerdo con lo que al res. pecto disponga la ley de procedimiento local" (Art. 7).

El Tratado regula también el cumplimiento de los actos procesales no contenciosos (Art. 10) y el libramiento de exhortos o cartas rogatorias que se refieran a embargos, tasaciones, inventarios o diligencias preventivas (Art. 12).

\section{2) Estado del Problema en el Uruguay.}

En nuestro país, independientemente de los tratados de Montevideo, existen disposiciones de nuestro Código de Procedimiento Civil en un capítulo titulado "Ejecución de las Sentencias dictadas por Tribunales Extranjeros" (incluído luego del de "De la Ejecución de las Sentencias"), cuyo origen es la ley de Enjuiciamiento Civil Española de 1855 (9). Son los Arts. 511 a 519, que otorgan competencia a la Suprema Corte de Justicia para conocer del juicio y estable. cen los requisitos comunes: Sentencia dictada por autoridad judicial competente, parte legalmente citada y representada en juicio o declarada rebelde, que no sea contraria a nuestra "orden, moral, constitución y leyes..." 
En el juicio de exequatur debe oirse a la contraparte y al Ministerio Público.

Se trata, como en el caso de los Tratados de Montevideo, de un juicio de reconocimiento tendiente a comprobar la existencia de los requisitos formales mencionados, efectuando una "declaración de certeza de la eficacia de la sentencia extranjera", como la denominara el maestro Carnelutti (10). La justicia nacional realiza un simple "juicio sumario de control" (11) que los italianos, en la práctica, han denominado "delibazione".

Nuestra doctrina ha discutido si este juicio de exequatur es necesario en todos los casos o solo en los que se pide el cumplimiento de la sentencia por medios ejecutivos (ejecución propiamente dicha). En particular se ha planteado el caso cuando la sentencia se presenta como prueba de un divorcio. Hay quienes han sostenido que aquí no se aplican estas disposiciones, sino las normas que rigen la presentación de documentos extranjeros (legalización) (12). Es la opinión de la cátedra de derecho internacional privado. En cambio generalmente los procesalistas sostuvieron, que aún en este caso, por tratarse de la eficacia de una sentencia extranjera, debe entender el máximo Tribunal, en el procedimiento indicado (13).

Nuestra jurisprudencia ha gido también oscilante. La Suprema Corte de Justicia entendió, hasta 1935, que sólo cabía su intervención en caso de ejecución propiamente dicha, no cuando se invocaba la sentencia como documento.

En 1935 se dictó una sentencia muy comentada que cambió este criterio sosteniendo, en un caso de divorcio, que esta sentencia no sólo vale como documento, sino que a ella se da fuerza ejecutoria al permitir la celebración de un nuevo acto que se efectúa en la República, o determinar la constitución de un nuevo estado civil (14).

Esta sentencia tuvo amplia repercusión diciendo los procesalistas que implicaba "la buena doctrina" (15) lo que no ha obstado para que en una décisión posterior fuera cambiada.

En efecto por sentencia dictada en el año 1953, resolviendo el exequatur de una decisión judicial dictada en Portugal, sostuvo que no se trata de la ejecución de una resolución de un Estado extranjero, sino de acreditar, el compareciente un estado civil, agregando que cuando se trata de prueba de un hecho o de una situación jurídica debe ser apreciada por la autoridad nacional ante la cual se invoque (16).

Se retoma, pues, la distinción entre ejecución de sentencia y presentación de ésta como documento, fundándose, especialmente, en las disposiciones de nuestro Código de Procedimiento, cuya letra se refiere a los actos propios de "ejecución" acordando la vía ejecutiva como proceso. 
Como vemos, pues, nuestra doctrina y jurisprudencia no son firmes, con respecto a este punto. En el resto coinciden en admitir la validez de la sentencia extranjera que se ejecutarú, previa apreciación del cumplimiento de los requisitos. formales ya expresados, según se trata de un país al cual nos liga o no el Tratado. En el primer caso será el Juez competente para el exequatur el que tenga competencia para la ejecución y no la Suprema Corte de Justicia.

\section{IV - PRINCIPIOS RECTORES}

Hemos querido exponer brevemente el problema y su solución en nuestro Derecho, pero no queremos terminar esta comunicación sin expresar algunos puntos de vista generalea, que pretenden servir de orientadores para llegar a alguna conclusión.

Aún cuando entremos brevemente en el tema de Derecho Internacional Privado, o sea el "por qué" se ejecuta una sentencia extranjera (17), creemos conveniente señalar los siguientes principios.

1. - Las relaciones internacionales han evolucionado de tal manera que el concepto de Soberanía del Estado ha entrado en crisis no solo frente a los grupos internos (gremios y otros grupos de presión) sino también frente al Derecho Internacional, no siendo hoy plenamente admitido el dogma de la soberanía absoluta.

Aunque vivimos en un Continente que por múltiples razones, especialmente de conveniencia política, sostiene el principio de la no intervención y auto-determinación de los pueblos, que está en la base de nuestro sistema de vida democrático, no debemos desconocer que la organización internacional progresa cada vez mas, y va limitando el campo de la soberanía nacional. Pertenecem todos los países aquí representados, a la Organización de las Naciones Unidas (ONU), integrada hoy con 105 Estados, organización que cuenta con un sistema jurídico, con órganos y con normas que le permite el empleo de la fuerza para imponerlas, aún contra la voluntad de los Estados. Pertenecemos muchos de los paísés aquí reunidos a la Organización de Estados Americanos (O.E.A.) que en la esfera de su competencia tiene una serie de atribuciones limitativas de la soberanía nacional.

Y todavía, independientemente de estos y otros organismos internacionales, aparece, empujada por el hecho económico, la creación de comunidades regionales, las cuales también, a sua vez, crean órganos que están por encima de la competencia nacional y modifican la realidad política y jurídica hasta ahora encerrada exclusivamente en el marco del Estado. Me refiero a la Comunidad Económica Europea y a la Asociación Latinoamericana de Libre Comercio.

No podríamos desconocer estos hechos que tienen una significación en sí, y además una trascendencia jurídica que nos está obli- 
gando a señalar que vivimos, sin perjuicio del Estado, en una comunidad internacional cuyas normas jurídicas son crecientes.

En consecuencia aparece vulnerado el principio que basaba todo el mecanismo de la ejecución de la sentencia extranjera en el siglo pasado, que consistía en reconocer a la sentencia solamente validez en el territorio en que es dictada, considerándola como un acto extranjero, al cual la soberanía de cada Estado admite mediante una aceptación previa y un juicio, que en algunos casos llega a revisar el proceso anterior en su forma y fondo.

Debemos limitar este derecho del Estado, emanado de su soberanía, negando la posibilidad de una revisión del fondo en cualquier caso.

Asimismo cabe rechazar el criterio de reciprocidad (18).

Hoy podemos hablar, sin duda, de un Juez competente en la esfera internacional $y$, por consiguiente, no de sentencias dictadas por Tribunales cuya autoridad debemos empezar por reconocer, sino por Organos cuya jurisdicción hemos aceptado de antemano para dictar normas que podrán ser ejecutadas en nuestro país.

$2 .^{\circ}$ - Frente a esta tendencia, debemos considerar, también, el principio de la soberanía nacional, que se mantiene indiscutiblemente a pesar de cierto debilitamiento. En su nombre el derecho internacional hace siempre la reserva de la jurisdicción doméstica. En nuestro tema se proclama el contralor (al menos formal) del cumplimiento de ciertos requisitos mínimos de la regularidad con que se llegó a la resolución que se pretende ejecutar, y la exigencia de que no contraríe el orden público del país donde se quiere cumplir.

Es otra realidad que los juristas debemos respetar, y de nada valdría aconsejar o resolver derrumbar esta valla. que hoy es infranqueable, aunque sea conveniente disminuir su altura cada vez mas.

3. - Por último queremos señalar que ese mínimo de contralor de la sentencia extranjera, para su ejecución por las autoridades nacionales, que se limita a apreciar ciertos elementos formales, representa la salvaguardia de los derechos de los individuos que están en juego.

En el fondo las reservas para la ejecución de la sentencia extranjera (cumplimiento de requisitos formales, debido emplazamiento, posibilidad de defenderse de la parte condenada, sentencia firme, etc.), representan otras tantas garantías para el cumplimiento de la sentencia misma. Es decir que el juicio de exequatur, en definitiva, no viene a ser una revisión del fallo (fondo del asunto), sino un contralor del cumplimiento de ciertos principios generales del contradictorio que, podemos decir, pertenecen a lo que se ha dado en llamar "las garantías del debido proceso" (19). 
En definitiva este contralor, en cuanto no signifique imiscuirse en la esfera del Tribunal que dictó la sentencia, ni revisar la misma, también responde a una necesidad de la organización actual y a las nuevas tendencias del Derecho Internacional que buscan, por encima de los derechos del Estado, la posibilidad de la defensa del propio individuo, a quien se considera como sujeto de Derecho Internacional luego de un largo proceso evolutivo (20).

Realmente al condicionar la ejecución de la sentencia al contralor del cumplimiento de los requisitos del debido proceso, en definitiva colocamos en primer plano al individuo, cuyo derecho subjetivo sacamos de la esfera de su propio Estado, para colocarlo bajo un nuevo control tutelar.

Estos principios podrían traducirse - en el tema de Homologación de Sentencia Extranjera - en las siguientes

\section{CONCLUSIONES:}

$\left.1 .^{\circ}\right)$ Es conveniente unificar los sistemas de ejecución de sentencia extranjera, colocando este tema entre uno de los fundamentales para la realización de acuerdos internacionales $y$, en lo que se refiere al continente americano, para la unificación de las normas de Derecho Procesal en el Continente.

2..$^{\circ}$ El Estado requerido para ejecutar una sentencia extranjera no debe entrar de ninguna manera a la revisión del fondo del asunto ni determinarse por la regla de reciprocidad.

$\left.3^{\circ}{ }^{\circ}\right)$ Frente al pedido de ejecución de una sentencia extranjera el Estado requerido deberá controlar, en juicio sumario de reconocimiento, que en la misma se hayan cumplido las garantías del debido proceso, especialmente: Competencia del Tribunal que la dictó, debida citación al demandado y oportunidad para defenderse y carácter definitivo del fallo.

4. $\left.{ }^{\circ}\right)$ Podrá dejarse de ejecutar la sentencia solamente si no cumple esos requisitos mínimos, o va contra el Orden Público del país requerido.

5..$\left.^{\circ}\right)$ Por ejecución de sentencia extranjera debe entenderse cualquier forma de eficacia de la misma, requiera o no medios compulsivos para su efectivo cumplimiento.

Misiones 1424. Piso $4 .^{\circ}$ Montevideo - Uruguay.

Montevideo, setiembre de 1962. 
(1) Especialmente luego del reciente libro publicado, entre nosotros, por Sentis Melendo, con su acostumbrada precisión, información y labor de síntesis ("La sentencia extranjera (Exequatur)". E.J.E.A. Buenos Aires 1958).

(2) Raúl Moretti. Revista Jurídica La Ley T. XV. Sec. Doct. p. $84-$ Rev. Derecho Público y Privado. T. 3 p. 142.

(3) Romero del Prado. La ley T. 50, pág. 964.

(4) Gelsi Bidart. Rev. de Derecho, Jurisprudencia y Administración. T. 56, págs. 236.

(5) Porque todavía, dentro de esitos casos cabrían otras distinciones. Así una parte de la doctrina Brasileña distingue dentro de una misma sentencia extranjera - de divorcio en cuanto a sua ejecución - dos variedades, las que son meramente declaratorios del estado civil de las personas, de aqueilas que requierem actos propios de ejecución. (Oscar Martin Gomes Sentenças estrangeiras de divorcio. Revista Forense 1960, N. 188, Año 57, pág. 438).

(6) Quintín Alfonsín. Ejecución extranacional de la sentencia. Rev. Der. Púb. y Priv. T. 26 págs. 195 y 259.

(7) Respecto de la ratificación de los tratados de 1889 y 1940 por los diversos países firmantes puede verse un cuadro en Vieira. Tratados de Montevideo. (Montevideo 1959).

(8) Actas de la Reunión de Jurisconsultos de Montevideo. 1939/40 N. ${ }^{\circ}$, p. 18.

(9) Rafael Gallinal. Estudios sobre el Código de Procedimiento Civil. Art. 511 y Sgts. pags. 185 y Sgts.

(10) Instituciones... E.J.E.A. Buenos Aires 1959. I, 69. Conforme Guasp. Derecho Procesal Civil. Madrid 1956 pags. 1316/17.

(11) Redenti. Diritto Processuale Civile. Milano. Giuffre 1957. III, 430.

(12) Alfonsín. La Justicia Uruguaya. T. 18 Sec. doct. p. 65.

(13) Moretti. Rev. La Ley. T. 15, Sec. doct. p. 84.

(14) C. A. Lazcano. Las sentencias extranjeras y el exequatur judicial. Jurisprudencia Argentina. T. 51. Sec. Doct. pag. 7.

(15) Lazcano. Ob. cit. jurisp. Arg. T. 51 doctr. pag. 9.

(16) Corresponde precisar que, en nuestro país, la organización del Registro del Estado Civil, desde que se estableció el matrimonio civil obligatorio, en el año 1885, fue encomendada a los Juzgados de Paz. Los jueces, de Paz, entonces, tienen una doble competencia: jurisdiccional en una serie de asuntos (especialmente los juicios menores de \$ 1.000) por lo cual dependen y son designados por la Suprema Corte de Justicia (Poder Judicial) y administrativa, como oficiales del Registro del Estado Civil que autorizan casamientos, e inscriben nacimientos y defunciones, 
dependientes del Ministerio de Instrucción Pública (Poder Ejecutivo). Por esa razón, en la función de oficiales del Estado Civil, dependen de un órgano ajeno a la Suprema Corte de Justicia. Esta decía, en la referida sentencia, que los órganos encargados de apreciar el documento, eran ajenos a su contralor.

(17) Sentis Melendo. Ob. cit. pag. 3, dice que el problema procesal no es el "por qué" sino el "como" de la ejecución.

(18) Este existe aún en muchos Códigos, como el uruguayo (art. 513) pero es rechazado por la doctrina (Theodore Sus. Rev. de Derecho Procesal. Madrid. 1952. pag. 3.

(19) Couture Estudios... T. 1, pag. 50 y Ss.

(20) Jacqueline Rochette L'individu devant le droit international. Paris 1956. Renée Cassin. L’homme sujet du droit international. "Etudes en honneur de G. Scelle pags. 67 y Ss. 